\title{
Observation of Superconductivity in Heavy-Fermion Compounds of $\mathrm{Ce}_{2} \mathrm{CoIn}_{8}$
}

\author{
Genfu Chen*, Shigeo Ohara, Masato Hedo ${ }^{1}$, Yoshiya Uwatoko ${ }^{1}$, \\ Kazuya $\mathrm{SAITO}^{2}$, Michio Sorai ${ }^{2}$ and Isao SAKAmoto \\ Department of Electrical and Computer Engineering, Nagoya Institute of Technology, Nagoya 466-8555 \\ ${ }^{1}$ The Institute for Solid State Physics, The University of Tokyo, Kashiwa 277-8581 \\ ${ }^{2}$ Research Center for Molecular Thermodynamics, Graduate School of Science, \\ Osaka University, Toyonaka, Osaka 560-0043 \\ (Received July 25, 2002; to be published December 2002)
}

\begin{abstract}
We succeeded in growing a single crystal of $\mathrm{Ce}_{2} \mathrm{CoIn}_{8}$ by the flux method. The results of specific heat and electrical resistivity measurements indicate that $\mathrm{Ce}_{2} \mathrm{CoIn}_{8}$ is a heavyfermion superconductor below $0.4 \mathrm{~K}$ with an electronic specific heat coefficient $\gamma$ as large as $500 \mathrm{~mJ} / \mathrm{K}^{2} \mathrm{~mol}-\mathrm{Ce}$.
\end{abstract}

KEYWORDS: superconductivity, heavy fermion, crystal growth, specific heat, electrical resistivity, $\mathrm{Ce}_{2} \mathrm{Coln}_{8}$

Considerable research has recently been focused on a new family of Ce-based heavy-fermion compounds with the general formula $\mathrm{Ce}_{n} M \mathrm{In}_{3 n+2}(M=\mathrm{Co}, \mathrm{Rh}, \mathrm{Ir} ; n=1$, 2 ), which crystallize in tetragonal $\mathrm{Ho}_{n} \mathrm{CoGa}_{3 n+2}$-type structures. ${ }^{1-3)}$ The crystal structure of $\mathrm{Ce}_{n} M \mathrm{In}_{3 n+2}$ can be seen as the $n$ layers of $\mathrm{CeIn}_{3}$ stacked sequentially along the $c$-axis with intervening one layer of $M \mathrm{In}_{2}$. These compounds exhibit a variety of interesting phenomena, such as heavy-fermion superconductivity, antiferromagnetism and pressure-induced superconductivity.

For transition metals $M=\mathrm{Rh}$ and Ir, a series of compounds $n=1$ and 2 has been synthesized in single crystal form. For $M=\mathrm{Rh}$, both $n=1$ and 2 compounds are antiferromagnets with Néel temperatures of $T_{N}=3.8$ and 2.8 $\mathrm{K}$ for $\mathrm{CeRhIn}_{5}$ and $\mathrm{Ce}_{2} \mathrm{RhIn}_{8}$, respectively. ${ }^{4,5)}$ The electronic specific heat coefficient $\gamma$ is estimated to be about $50 \mathrm{~mJ} / \mathrm{K}^{2} \mathrm{~mol}-\mathrm{Ce}$ for $\mathrm{CeRhIn}_{5}$ and $370 \mathrm{~mJ} / \mathrm{K}^{2} \mathrm{~mol}-\mathrm{Ce}$ for $\mathrm{Ce}_{2} \mathrm{RhIn}_{8} \cdot{ }^{6,7)}$ In both compounds, superconductivity was observed under high pressures. $\mathrm{CeRhIn}_{5}$ transforms from antiferromagnetic state to superconducting state at $2 \mathrm{~K}$ under the pressure of $1.6 \mathrm{GPa}^{4}{ }^{4}$ For $\mathrm{Ce}_{2} \mathrm{RhIn}_{8}$, very recently, pressure-induced superconductivity was reported. ${ }^{8)}$

For $M=\mathrm{Ir}$, the $n=1$ member $\mathrm{CeIrIn}_{5}$ is a heavy-fermion superconductor with a transition temperature of $T_{c}=0.4$ $\mathrm{K}$ at ambient pressure, while the $n=2$ member $\mathrm{Ce}_{2} \operatorname{IrIn}_{8}$ is a paramagnet down to $50 \mathrm{mK}$ with no evidence of a phase transition. ${ }^{5,9)}$ The $\gamma$ values are about $700 \mathrm{~mJ} / \mathrm{K}^{2} \mathrm{~mol}-\mathrm{Ce}$ for both compounds. ${ }^{5,10)}$

For $M=\mathrm{Co}$, the $n=1$ member $\mathrm{CeCoIn}_{5}$ is a heavyfermion superconductor with a transition temperature of $T_{c}=2.3 \mathrm{~K}^{11)}$ The $\gamma$ value of $\mathrm{CeCoIn}_{5}$ is about 300 $\mathrm{mJ} / \mathrm{K}^{2} \mathrm{~mol}-\mathrm{Ce}$ at $T_{c}$. When the superconductivity is suppressed by a magnetic field of $50 \mathrm{kOe}$, the $\gamma$ value increases with decreasing temperature and reaches a very large value of about $1 \mathrm{~J} / \mathrm{K}^{2}$ mol-Ce. ${ }^{11,12)}$ To our knowledge, however, there is no report on the $n=2$ member $\mathrm{Ce}_{2} \mathrm{CoIn}_{8}$, except for the structural study. ${ }^{1,2)}$

The purpose of this paper is to clarify the thermal

* Present address: Department of Electrical and Computer Engineering, Nagoya Institute of Technology, Nagoya 466-8555 and electronic properties of $\mathrm{Ce}_{2} \mathrm{CoIn}_{8}$. We attempted to synthesize $\mathrm{Ce}_{2} \mathrm{CoIn}_{8}$ by the flux method and found the conditions for single crystal growth. Measurements of specific heat and electrical resistivity were carried out on single crystals of $\mathrm{Ce}_{2} \mathrm{CoIn}_{8}$. In this paper, we report the observed superconductivity at $T_{c} \sim 0.4 \mathrm{~K}$ for $\mathrm{Ce}_{2} \mathrm{CoIn}_{8}$ at ambient pressure.

Single crystals of $\mathrm{Ce}_{2} \mathrm{CoIn}_{8}$ were grown from an In flux. An arc-melted $\mathrm{Ce}_{2} \mathrm{Co}$ button and excess In were sealed in a quartz ampoule under high vacuum. The ampoule was heated up to $750^{\circ} \mathrm{C}$ over $7 \mathrm{~h}$ and then raised quickly to $1000^{\circ} \mathrm{C}$ and held for 5 minutes, followed by rapid cooling to $750^{\circ} \mathrm{C}$. The single crystals grew in the temperature region of $750-300^{\circ} \mathrm{C}$. After spinning off the remaining In, many mm-sized, cube(or tetragon)-withoctahedron-like single crystals were obtained. The residual In on grown crystal surfaces was further removed by acid etching. The crystals were characterized by a powder X-ray diffraction method using $\mathrm{Cu} K \alpha$ radiation at room temperature. The specific heat was measured by the relaxation method in the temperature range of 2 $20 \mathrm{~K}$. The resistivity measurement was performed by a standard four-probe ac bridge method down to $0.1 \mathrm{~K}$.

Figure 1 shows the X-ray diffraction pattern obtained for crushed crystals. The X-ray pattern is well indexed on the base of the tetragonal $\mathrm{Ho}_{2} \mathrm{CoGa}_{8}$-type structure (the space group $\mathrm{P} 4 / \mathrm{mmm}$ ), as indicated by the indices in Fig. 1. There is no trace of $\mathrm{CeCoIn}_{5}$. A weak peak, marked by an arrow, is the (011) reflection of the residual In-flux. The lattice parameters are determined as $a=$ $4.643 \AA$ and $c=12.25 \AA$, which agree with the reported values. ${ }^{1)}$

Specific heat divided by temperature $C_{p} / T$ vs $T^{2}$ for $\mathrm{Ce}_{2} \mathrm{CoIn}_{8}$ is illustrated in Fig. 2. With decreasing temperature, $C_{p} / T$ decreases linearly for $T^{2}$ and shows a minimum around $7 \mathrm{~K}$. At lower temperatures, $C_{p} / T$ increases with decreasing temperature. At $2 \mathrm{~K}, C_{p} / T$ reaches $500 \mathrm{~mJ} / \mathrm{K}^{2} \mathrm{~mol}-\mathrm{Ce}$. This indicates that the $\gamma$ value of $\mathrm{Ce}_{2} \mathrm{CoIn}_{8}$ is more than $500 \mathrm{~mJ} / \mathrm{K}^{2} \mathrm{~mol}-\mathrm{Ce}$, showing a heavy-fermion characteristic.

Figure 3 shows the temperature dependence of the 


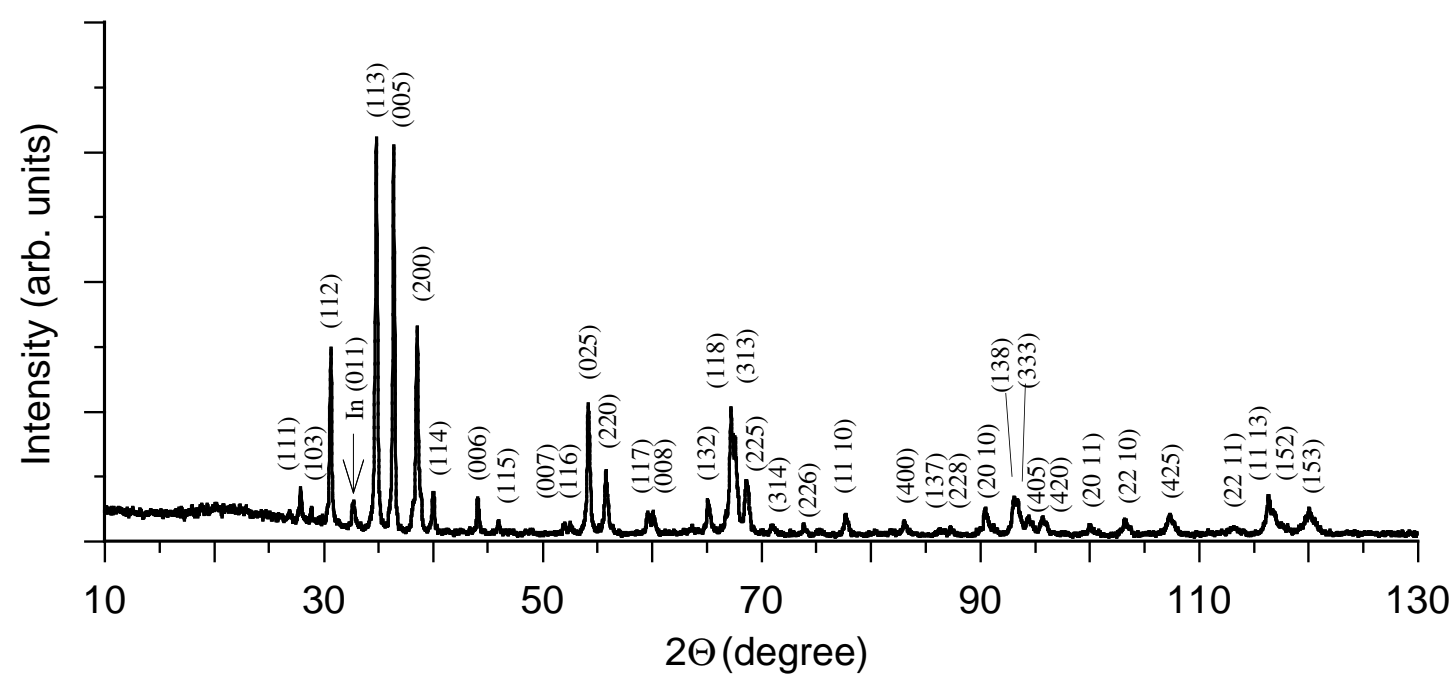

Fig. 1. X-ray diffraction pattern of $\mathrm{Ce}_{2} \mathrm{CoIn}_{8}$ with Miller indices. The arrow denotes the (011) reflection of the residual In-flux.

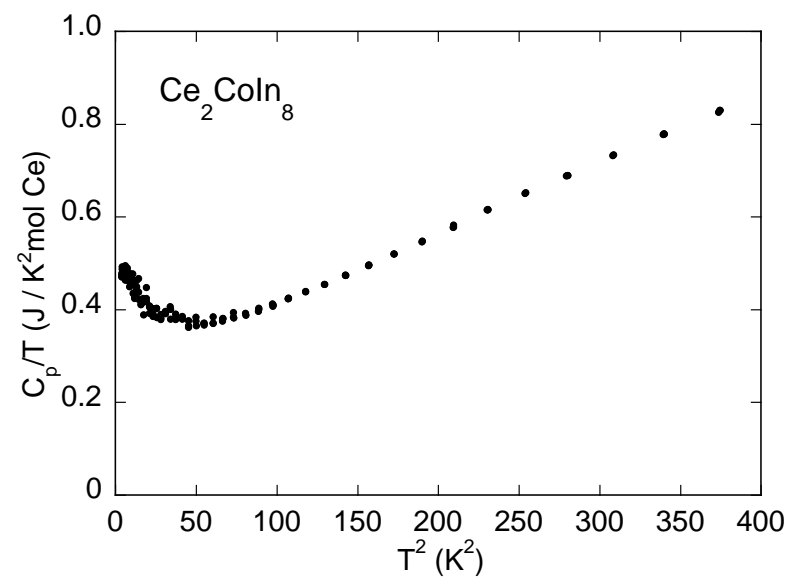

Fig. 2. Specific heat divided by temperature $C_{p} / T$ versus $T^{2}$ for $\mathrm{Ce}_{2} \mathrm{CoIn}_{8}$.

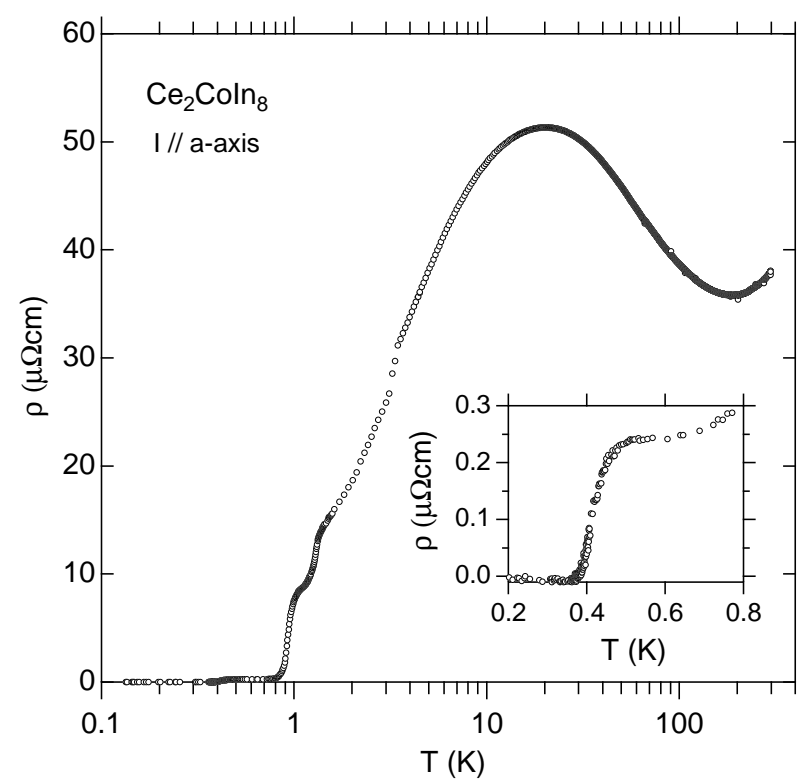

Fig. 3. Electrical resistivity plotted with $\log T$ in the temperature range from 0.1 to $300 \mathrm{~K}$ for $\mathrm{Ce}_{2} \mathrm{CoIn}_{8}$. The inset shows the resistivity in the lowest temperature region on a linear scale. electrical resistivity $\rho$ as a function of $\log T$ with the current along the $a$-axis. The inset shows the resistivity below $0.8 \mathrm{~K}$ on a linear scale. With decreasing temperature, the resistivity shows a $-\log T$ dependence below $80 \mathrm{~K}$ down to $30 \mathrm{~K}$, reaches a maximum, and decreases at lower temperatures. A kink at $3.4 \mathrm{~K}$ is attributed to the superconducting transition of free In that might be included in the crystal. As the temperature decreases further, the $\rho$ - $T$ curve shows a few abrupt drops near $1.4 \mathrm{~K}, 1.0 \mathrm{~K}$ and $0.4 \mathrm{~K}$, as shown in Fig. 3. Below 0.4 $\mathrm{K}$, the resistivity becomes zero, as shown in the inset in Fig. 3. This suggests that $\mathrm{Ce}_{2} \mathrm{CoIn}_{8}$ is a heavy-fermion superconductor with a transition temperature of $T_{c} \sim 0.4$ K.

To determine the upper critical field $H_{c 2}$ for $\mathrm{Ce}_{2} \mathrm{CoIn}_{8}$, we measured the resistivity on a single crystal $\mathrm{Ce}_{2} \mathrm{CoIn}_{8}$ with a magnetic field applied along the $a$-axis. The zero resistivity state is broken with the field $H=1 \mathrm{~T}$ at $0.1 \mathrm{~K}$, which indicates that $H_{c 2} \sim 1 \mathrm{~T}$ for $\mathrm{Ce}_{2} \mathrm{CoIn}_{8}$. The values of $T_{c} \sim 0.4 \mathrm{~K}$ and $H_{c 2} \sim 1 \mathrm{~T}$ for $\mathrm{Ce}_{2} \mathrm{CoIn}_{8}$ are much lower than those for $\mathrm{CeCoIn}_{5}$, but are very similar to those for CeIrIn $\left._{5} \cdot{ }^{7}, 13,14\right)$ The complete $H$ - $T$ phase diagram will be reported in the near future.

The causes of the resistivity drops near $T=1.4$ and $1.0 \mathrm{~K}$ for $\mathrm{Ce}_{2} \mathrm{CoIn}_{8}$ are yet to be clarified. However, a similar resistivity anomaly is also observed in $\mathrm{CeIrIn}_{5}$, where the anomaly is viewed as an appearance of superconductivity but is distinguished from bulk superconductivity. ${ }^{7,9,15)}$ To clarify the origin of these resistivity drops and the bulk properties of the superconducting state for $\mathrm{Ce}_{2} \mathrm{CoIn}_{8}$, more detailed studies of specific heat and magnetic susceptibility at lower temperatures are being carried out.

Here, we have presented the specific heat and the resistivity measurements performed on the single crystals of $\mathrm{Ce}_{2} \mathrm{CoIn}_{8}$. It is found that $\mathrm{Ce}_{2} \mathrm{CoIn}_{8}$ is a heavy-fermion superconductor with $T_{c} \sim 0.4 \mathrm{~K}$.

We thank S. Okada, Y. Shomi and Y. X. Jin for their experimental assistance. One of the authors (S. Ohara) was financially supported by the Nitto Foundation. 
1) Ya. M. Kalychak, V. I. Zaremba, V. M. Baranyak, V. A. Bruskov and P. Yu. Zavalij: Izv. Acad. Nauk SSSR Metally 1 (1989) 209.

2) Ya. M. Kalychak: J. Alloys compd. 291 (1999) 80.

3) E. G. Moshopoulou, Z. Fisk, J. L. Sarrao and J. D. Thompson: J. Solid State Chem. 158 (2001) 25.

4) H. Hegger, C. Petrovic, E. G. Moshopoulou, M. F. Hundley, J. L. Sarrao, Z. Fisk and J. D. Thompson: Phys. Rev. Lett. $84(2000) 4986$.

5) J. D. Thompson, R. Movshovich, Z. Fisk, F. Bouquet, N. J. Curro, R. A. Fisher, P. C. Hammel, H. Hegger, M. F. Hundley, M. Jaime, P. G. Pagliuso, C. Petrovic, N. E. Phillips and J. L. Sarrao: J. Magn. \& Magn. Mater. 226 (2001) 5.

6) P. G. Pagliuso, M. F. Hundley and J. L. Sarrao: Phys. Rev. B 64 (2001) 144411.

7) H. Shishido, R. Settai, D. Aoki, S. Ikeda, H. Nakazawa, N. Nakamura, T. Iizuka, Y. Inada, K. Sugiyama, T. Takeuchi, K. Kindo, T. C. Kobayashi, Y. Haga, H. Harima, Y. Aoki, T. Namiki, H. Sato and Y. Onuki: J. Phys. Soc. Jpn. 71 (2002) 162.

8) M. Nicklas, V. A. Sidorov, H. A. Borges, P. G. Pagliuso, Z.
Fisk, J. L. Sarrao and J. D. Thompson: cond-mat/0204064.

9) C. Petrovic, R. Movshovich, M. Jaime, P. G. Pagliuso, M. F. Hundley, J. L. Sarrao, Z. Fisk and J. D. Thompson: Europhysics Lett. 53 (2001) 354.

10) J. S. Kim, J. Alwood, G. R. Stewart, J. L. Sarrao and J. D. Thompson: Phys. Rev. B 64 (2001) 134524.

11) C. Petrovic, P. G. Pagliuso, M. F. Hundley, R. Movshovich, J. L. Sarrao, J. D. Thompson, Z. Fisk and P. Monthoux: J. Phys. Condens. Matter 13 (2001) L337.

12) S. Ikeda, H. Shishido, M. Nakashima, R. Settai, D. Aoki, Y. Haga, H. Harima, Y. Aoki, T. Namiki, H. Sato and Y. Onuki: J. Phys. Soc. Jpn. 70 (2001) 2248.

13) R. Movshovich, A. Bianchi, M. Jaime, M. F. Hundley, J. D. Thompson, N. J. Curro, P. C. Hammel, Z. Fisk, P. G. Pagliuso and J. L. Sarrao: Physica B 312-313 (2002) 7.

14) G. Sparn, R. Borth, E. Lengyel, P. G. Pagliuso, J. L. Sarrao, F. Steglich, M. Jaime and J. D. Thompson: Physica B 319 (2002) 262.

15) A. Bianchi, R. Movshovich, M. Jaime, J. D. Thompson, P. G. Pagliuso and J. L. Sarrao: Phys. Rev. B 64 (2001) 220504(R). 